\title{
Phosphatidylcholine-Bound Silybin
}

National Cancer Institute

\section{Source}

National Cancer Institute. Phosphatidylcholine-Bound Silybin. NCI Thesaurus. Code C71883.

An oral preparation of the flavonoid silybin with potential antioxidant and chemopreventive activities. Silybin, also known as silibinin, is a mixture of two stereoisomers, denoted silybin A and silybin B, and is the major active constituent of silymarin, a mixture of flavonolignans extracted from blessed milk thistle (Silybum marianum). Silybin modulates P-glycoprotein (P-gp)-mediated cellular efflux; has oxygen radical-scavenging effects; inhibits the arachidonic acid pathway; and inhibits various cytochrome P450 enzymes. This agent may also exhibit anti-angiogenic activity, possibly by inducing endothelial cell apoptosis via modulation of the transcription factor NF-kB, the Bcl-2 family of proteins, and caspases. Complexing silybin with phosphatidylcholine increases its bioavailability. 